Research Article

\title{
Experimental and Numerical Investigation on the Effects of Foundation Pit Excavation on Adjacent Tunnels in Soft Soil
}

\author{
Zi-Tian Yu, ${ }^{1,2,3}$ Heng-Yu Wang ${ }^{(D)}{ }^{2,3,4}$ Wenjun Wang, ${ }^{2,4}$ Dao-Sheng Ling, ${ }^{1,2,3}$ \\ Xue-Dong Zhang, ${ }^{5}$ Can Wang, ${ }^{6}$ and Yong-Hao $\mathrm{Qu}^{7}$ \\ ${ }^{1}$ Institute of Geotechnical Engineering, Zhejiang University, Hangzhou 310058, China \\ ${ }^{2}$ School of Civil Engineering and Architecture, NingboTech University, Ningbo 315100, China \\ ${ }^{3}$ MOE Key Laboratory of Soft Soils and Geoenvironmental Engineering, Zhejiang University, Hangzhou 310058, China \\ ${ }^{4}$ Ningbo Research Institute, Zhejiang University, Ningbo 315100, China \\ ${ }^{5}$ Department of Geotechnical Engineering, China Institute of Water Resources and Hydropower Research, Beijing 100048, China \\ ${ }^{6}$ China Harbour Engineering Company LTD, Beijing 100027, China \\ ${ }^{7}$ China Railway Construction Bridge Engineering Bureau Group Company LTD, Tianjin 300300, China
}

Correspondence should be addressed to Heng-Yu Wang; 10912019@zju.edu.cn

Received 10 February 2021; Revised 27 March 2021; Accepted 13 April 2021; Published 22 April 2021

Academic Editor: Wangcheng Zhang

Copyright (c) $2021 \mathrm{Zi}$-Tian Yu et al. This is an open access article distributed under the Creative Commons Attribution License, which permits unrestricted use, distribution, and reproduction in any medium, provided the original work is properly cited.

Excavations near an existing tunnel are often encountered in underground construction. The influence of the excavation on the adjacent tunnels is not yet fully understood. This study presented a centrifugal model test about excavation next to existing tunnels in soft soil foundation. The bending moment of diaphragm wall, surface settlement, tunnel deformation, and earth pressure around the tunnel were mainly studied. The influence of tunnel location is further studied by numerical simulation. During the stabilization stage of foundation pit, the diaphragm walls present convex deformation towards foundation pit, and the surface settlement outside the diaphragm wall appears to be the concave groove type. During the overexcavation stage, the diaphragm walls are almost damaged, and the shear bands are nearly tangent to the tunnels. The displacement of the tunnels and the surface settlement rapidly increase. The deformation of the diaphragm wall and the surface settlement are limited by the existing tunnel. The numerical results indicate that the change of tunnel location has little effect on the retaining wall but an obvious effect on the tunnel itself.

\section{Introduction}

With the rapid development of the construction of coastal cities, the excavation of foundation pit is often close to the existing tunnels. The foundation pit excavation in coastal soft soil can lead to soil unloading. Then additional stress and deformation to the adjacent existing subway tunnels may be formed due to the change of the stress field and soil displacement [1]. Since the coastal soft soil is generally characterized by low strength, high moisture content, and high compressibility, it will be easier to be weakened due to the construction disturbance, which further complicates the engineering problems [2].

Many researchers have studied the effect of tunnel location during excavation. Zheng and Wei [3] carried out a numerical parametric study to investigate tunnel deformation and stress redistribution around the tunnel due to basement excavation. They found that the tunnel deformation mode is influenced by the distance between the tunnel and the diaphragm wall. Huang et al. [4] studied the influence of the relative location of tunnel with respect to the excavation, tunnel diameter, and excavation dimensions through numerical analysis. $\mathrm{Ng}$ et al. [5, 6] analyzed the effect of the tunnel location on the internal force and deformation of the tunnel during the excavation. With the development of centrifugal model tests in geotechnical engineering, many researchers have performed centrifugal model tests about the foundation pit excavation. Centrifugal model test is the most advanced and effective test method in geotechnical engineering, which can reproduce the field conditions, notably the stress field. In dry sand foundation, 
many centrifugal model tests have been carried out to study the effect of excavation on the existing tunnel [5-9]. It should be noted that the behavior of tunnels and diaphragm walls in saturated clay is generally different from that in dry sand. But few relevant studies have been reported on the current literature, especially the study of the relative location between the tunnel and excavation.

In this study, a centrifugal model test in soft soil foundation was conducted to evaluate the influences of the excavation on the existing tunnel, nearby ground, and diaphragm wall. This paper aims to obtain the deformation and stress characteristics of the tunnels and study the variation law of the deformation and bending moment of the diaphragm walls, the surface settlement outside the foundation pit, the earth pressure, and so forth. The influence of the excavation in soft soil on the tunnels in different location is revealed, and the condition of overexcavation is simulated. Besides, the experimental data are compared with the numerical results obtained from the finite element method to demonstrate the validity of the numerical method and the corresponding model. Numerical analysis is performed to investigate the effect of the tunnel space location on the tunnels and diaphragm walls.

\section{Centrifugal Model Test}

The experiment was performed on the LXJ-4-450 geotechnical centrifuge at China Institute of Water Resources and Hydropower Research. The centrifuge has a payload of $1.5 \mathrm{t}$, a maximum rotation radius of $5.03 \mathrm{~m}$, a maximum acceleration of $300 \mathrm{~g}$, and a payload capacity of $450 \mathrm{~g}$-t. The size of the model test box is $1.32 \mathrm{~m} \times 0.62 \mathrm{~m} \times 0.8 \mathrm{~m}$.

2.1. The Test Material. The sandy kaolin was used as the soil foundation in this centrifugal model test. The basic physical parameters of sandy kaolin are shown in Table 1. In the process of the soil foundation preparation, the kaolin powder was mixed with 1.5 times water of liquid limit to make mud in an agitator, and then the saturated kaolin foundation was consolidated under self-weight using the centrifugal model test.

2.2. The Centrifugal Model. Considering the model box size, tunnel size, foundation pit width, excavation depth, and the influence range of excavation in soft soil foundation [10], the centrifugal acceleration in the experiment was chosen as $75 \mathrm{~g}$. The plan and section of the arrangement of each component are shown in Figure 1. The diaphragm walls and tunnel in the centrifugal model test are designed by the method of equivalent elastic modulus [5-9]. As shown in Figure 2, the diaphragm walls, which are made of the aluminum plate, are equivalent to an $800 \mathrm{~mm}$ thick concrete diaphragm wall with an elastic modulus of $70 \mathrm{GPa}$. Based on the principle of equivalent compressive stiffness, the horizontal bracing system, which is made of the aluminum bar, is equivalent to steel support with a diameter of $0.609 \mathrm{~m}$. The inner supports are connected with the diaphragm walls by bolts. Only one layer of the inner supports is set up on the
TABLE 1: Physical parameters of sandy kaolin.

\begin{tabular}{lccccc}
\hline$G_{\mathrm{s}}$ & $W_{\mathrm{l}}(\%)$ & $I_{\mathrm{p}}(\%)$ & $C_{\mathrm{v}}\left(\mathrm{m}^{2} /\right.$ year $)$ & $c^{\prime}(\mathrm{kPa})$ & $\varphi^{\prime}\left(^{\circ}\right)$ \\
\hline 2.67 & 62.2 & 32.0 & 85 & 2.67 & 23 \\
\hline
\end{tabular}

upper part of the diaphragm walls to simulate the overexcavation condition.

As shown in Figure 3, based on the equivalent elastic modulus, the tunnel, which is made of the aluminum tube, is equivalent to a reinforced concrete-lined tunnel with an elastic modulus of $35 \mathrm{GPa}$. The model tunnel had an outer diameter of $80 \mathrm{~mm}$ (6 $\mathrm{m}$ in prototype) and thickness of $3.0 \mathrm{~mm}(0.225 \mathrm{~m}$ in prototype).

The excavation depth was $120 \mathrm{~mm}$ ( $9 \mathrm{~m}$ in prototype). The diaphragm walls were $210 \mathrm{~mm}$ high $(15.75 \mathrm{~m}$ in prototype), and hence the penetration depth below the final excavation depth was $90 \mathrm{~mm}$ ( $6.75 \mathrm{~m}$ in prototype). The tunnels were placed on both sides of the foundation pit, which are $80 \mathrm{~mm}$ and $120 \mathrm{~mm}$ ( $9 \mathrm{~m}$ and $12 \mathrm{~m}$ in prototype) away from the diaphragm wall, respectively. The depth of foundation soil is $300 \mathrm{~mm}$ (22.5 $\mathrm{m}$ in prototype). The model and prototype sizes are listed in Table 2.

2.3. The Sensors Arrangement. In the centrifugal model test, the internal force and deformation characteristics of the diaphragm walls, the surface settlement outside the foundation pit, and the force and deformation characteristics of the tunnels during the foundation pit excavation were monitored.

As shown in Figure 1, the strain gauges were vertically pasted along the middle of the diaphragm wall. Due to the limitation of test conditions, the strain gauges were configured to form a $1 / 4$ bridge circuit. Two laser displacement meters were arranged on top of the diaphragm walls to monitor the horizontal displacement of the top of the diaphragm walls. Six laser displacement meters were arranged at $0.5 \mathrm{H}_{\mathrm{e}}, \mathrm{H}_{\mathrm{e}}$, and $2 \mathrm{H}_{\mathrm{e}}$ away from the diaphragm wall, respectively, to monitor the ground surface settlement $\left(\mathrm{H}_{\mathrm{e}}\right.$ is the excavation depth of the foundation pit). A digital camera was set up on the long side of the model box to record the deformation and displacement of the foundation pit and tunnels during the whole experiment. The monitoring locations of the hoop strain were in the middle of the longitudinal direction of the tunnels. The strain gauges were arranged every $45^{\circ}$ along the circle of the tunnels using the method of $1 / 4$ bridge. The miniature earth pressure gauges were located at $1 \mathrm{D}$ away from the middle of the longitudinal direction of the tunnels ( $D$ is the diameter of the tunnel) and arranged every $90^{\circ}$ along the circle of the tunnels.

2.4. The Test Procedure. Kaolin was mixed with 1.5 times liquid limit water to prepare slurry and then poured into the model box for stratified static consolidation. In order to make the tunnel and soil displacement clearer, kaolin was poured in layers along with colored sand. When the soil was poured and consolidated in layers, the tunnels were placed in the appropriate place. After the soft soil of sandy kaolin was 


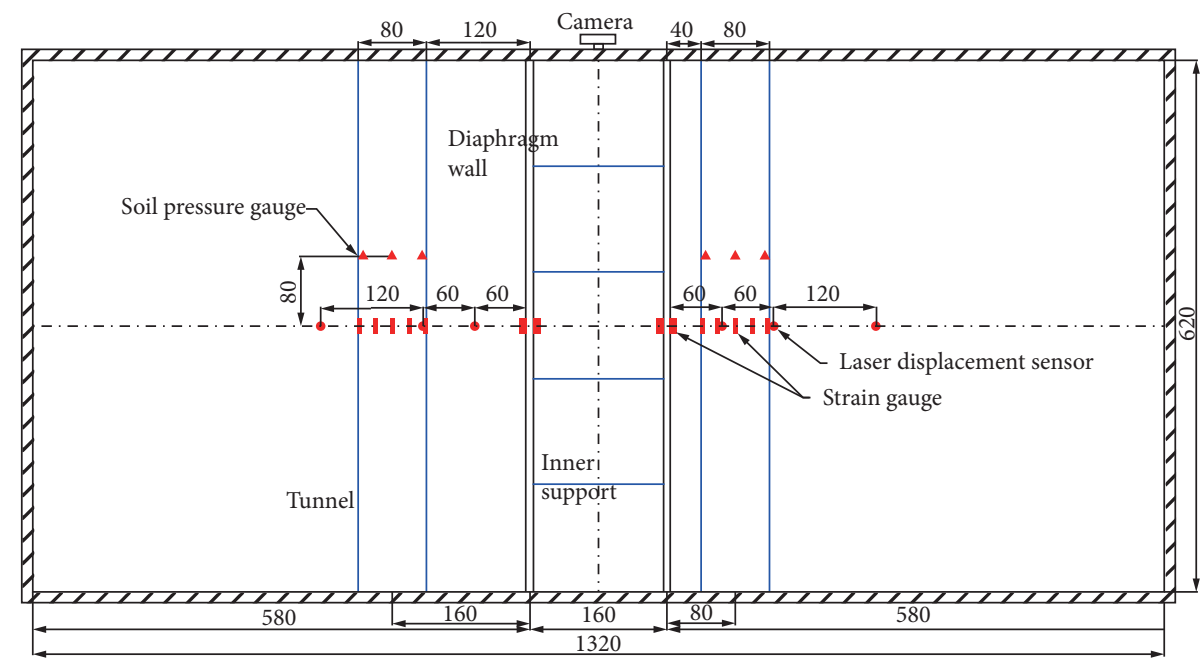

(a)

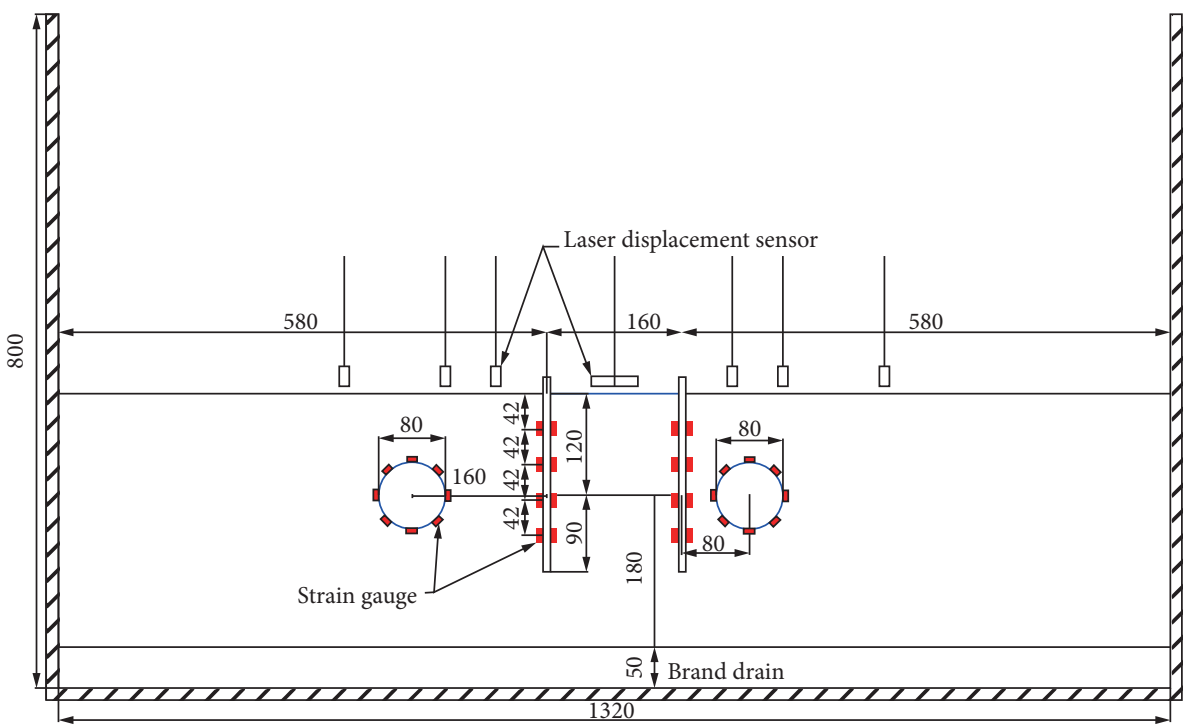

(b)

FIGURE 1: Schematic of centrifuge model (unit: $\mathrm{mm}$ ). (a) Plan of the arrangement of each component. (b) Section of the arrangement of each component.

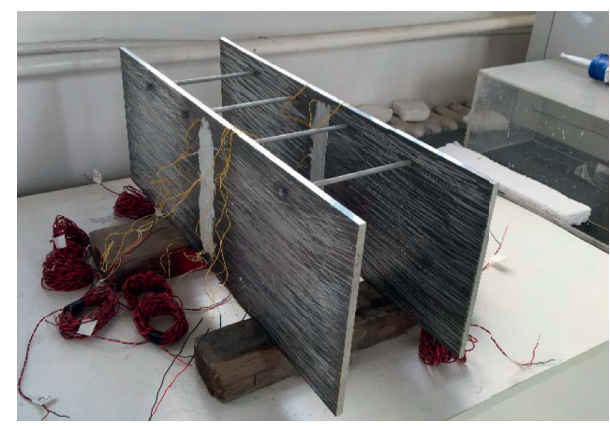

FIGURE 2: Picture of the diaphragm walls and inner supports model.

consolidated, the diaphragm walls and inner supports model was inserted into the foundation soil. The centrifuge acceleration was raised up to $75 \mathrm{~g}$ and remained stable for 10 minutes. The initial data of each sensor were obtained

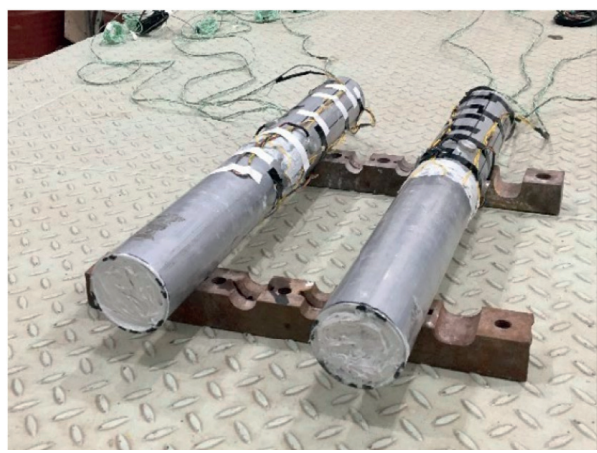

FIgURE 3: Picture of the tunnel models.

when the centrifuge acceleration remained stable. After the centrifuge acceleration was reduced to $0 \mathrm{~g}$, the soil in the foundation pit was excavated with a depth of $40 \mathrm{~mm}$. The 
TABLe 2: Model and prototype sizes.

\begin{tabular}{|c|c|c|c|}
\hline & & Prototype $(\mathrm{m})$ & Model (mm) \\
\hline \multirow{5}{*}{ Diaphragm wall } & Width of the foundation pit & 12 & 160 \\
\hline & Excavation depth & 9 & 120 \\
\hline & Insert depth of the diaphragm wall & 6.75 & 90 \\
\hline & The thickness of the diaphragm wall & 0.8 & 8 \\
\hline & Diameter of the inner support & $\Phi 0.61$ & $\Phi 4.5$ \\
\hline \multirow{4}{*}{ Tunnel } & Diameter & 6 & 80 \\
\hline & The thickness of the lining & 0.225 & 3 \\
\hline & Distance between the tunnel and diaphragm wall & 6 or 12 & 80 or 160 \\
\hline & Buried depth & 9 & 120 \\
\hline
\end{tabular}

centrifuge acceleration was raised up to $75 \mathrm{~g}$ again, and the data of each sensor were obtained when the centrifuge acceleration remained stable. The foundation pit was excavated to the bottom in three stages, and the excavation depth of each stage was $40 \mathrm{~mm}$ ( $3 \mathrm{~m}$ in prototype). The total excavation depth of the foundation pit was $120 \mathrm{~mm}(9 \mathrm{~m}$ in prototype).

\section{Results and Discussion}

The following results of the centrifugal model test are converted into the corresponding values of the prototype for convenience.

3.1. The Development of the Shear Band. As shown in Figure 4, in the first and second excavation, the deformation of the diaphragm walls and the settlement of the ground surface are small, and the foundation pit remains stable. But a rapid increase in the deformation of the diaphragm walls and the settlement of the ground surface appears in the third stage of excavation due to the fact that only one layer of the inner supports is set up on the upper part of the diaphragm walls. In the third stage, the bottom of the foundation pit heaves severely, and the diaphragm walls lose stability finally. As shown in Figure 4(b), kick-in type failure occurred to the diaphragm walls. The curve obtained by connecting the crack point of the ground surface, the bottom of the diaphragm walls, and the inflection points of the colored sand layers is the shear band for the case of the instability failure of the foundation pit [11]. Figure 4 shows that both shear bands pass through the points that are near the arch waist of the tunnel and are nearly tangent to the tunnel. The shear band, which is on the far side of the tunnel from the diaphragm wall, spreads wider than on the other side. It is indicated that the tunnel location will affect the formation of the shear band in the soil.

3.2. The Bending Moment of the Diaphragm Walls. The bending moment of the diaphragm walls along the depth at the different excavation steps is shown in Figure 5. The depth is normalized, and $\mathrm{H}_{\mathrm{e}}$ is the maximum excavation depth. The bending moment towards the inside of the foundation pit is recorded as a positive value. It is shown that the bending moment of the diaphragm walls on both sides gradually increases with the increase of the excavation depth. After the first and second excavation, the bending moments of the diaphragm walls on the left and right sides approach each other. Diaphragm walls present convex deformation towards foundation pit. The bending moment on both sides is relatively small at both ends of the diaphragm walls, and the maximum bending moment of the diaphragm walls is located about 0.7 times the excavation depth. After the third excavation, the bending moment form of the diaphragm wall which is far away from the tunnel changes. The maximum bending moment is located at the bottom of the diaphragm wall, and the maximum bending moment is 1.55 times higher than that of the previous step excavation. The diaphragm wall, which is far away from the tunnel, is almost damaged. The bending moment form of the diaphragm wall near the tunnel still appears to be convex, and the maximum bending moment increases by $45 \%$ compared with that for the previous excavation. These results suggest that the deformation of the soil and diaphragm wall is limited by the existing tunnel.

3.3. The Horizontal Displacement of the Diaphragm Walls. Figure 6 shows the curves of the horizontal displacement of the diaphragm walls along the depth at the different excavation steps. The horizontal displacement of the diaphragm walls is normalized. Since the foundation pit is damaged after the third excavation and the measurement for the displacement of the diaphragm wall is only appropriate for the small deformation, the horizontal displacement of the diaphragm walls after the first and second excavation is analyzed.

As is shown, the displacement of the left and right diaphragm walls gradually increases with the increase of the excavation depth. The difference in the horizontal displacement between two diaphragm walls is not significant, and both the diaphragm walls present convex deformation. This deformation type is similar to that in Xu's study [12]. After the first and second excavation, the maximum displacement of the diaphragm walls is located about 0.7 times the excavation depth. The maximum displacement of the diaphragm walls on both sides is about $0.022 \%$ of the excavation depth after the first excavation, and, after the second excavation, the maximum displacement of the diaphragm walls on both sides is about $0.058 \%$ of the excavation depth. It can be seen that the tunnel location has little influence on the deformation of the diaphragm wall in this test during the first and second excavation. 


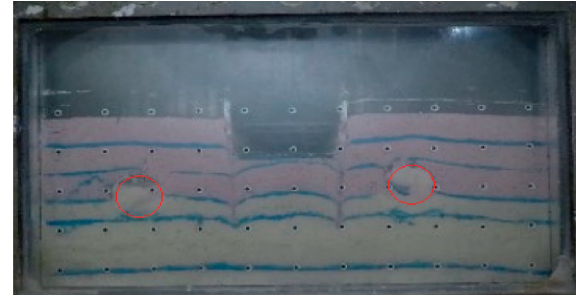

(a)

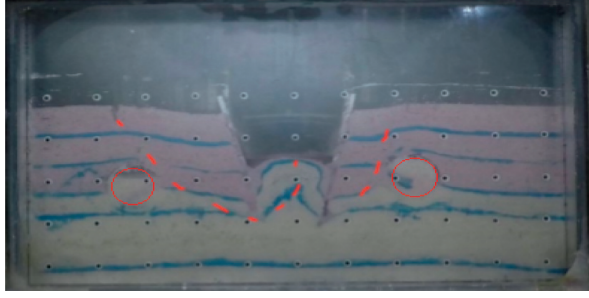

(b)

Figure 4: Elevation view of experiment phenomena. (a) Stable phase. (b) Failure phase.

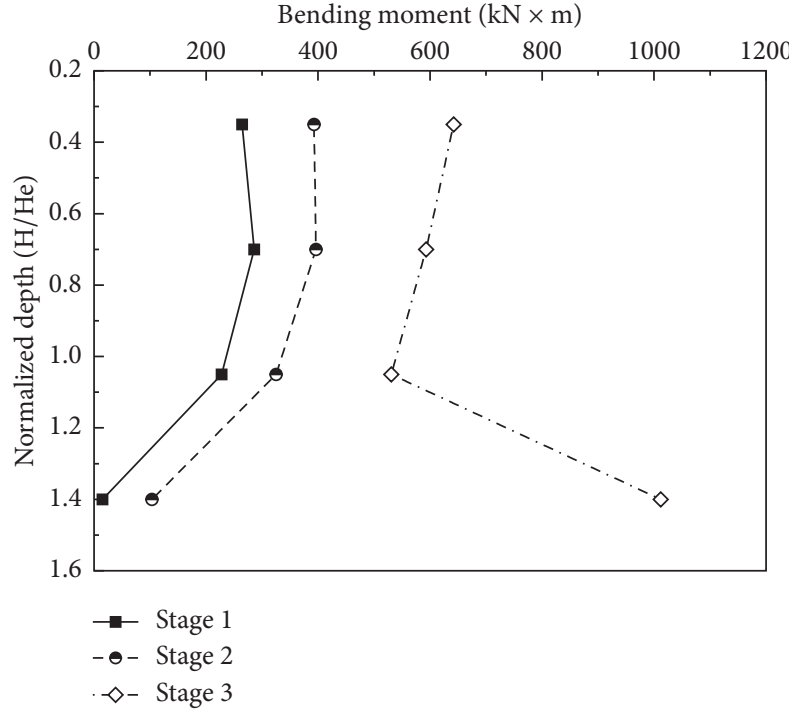

(a)

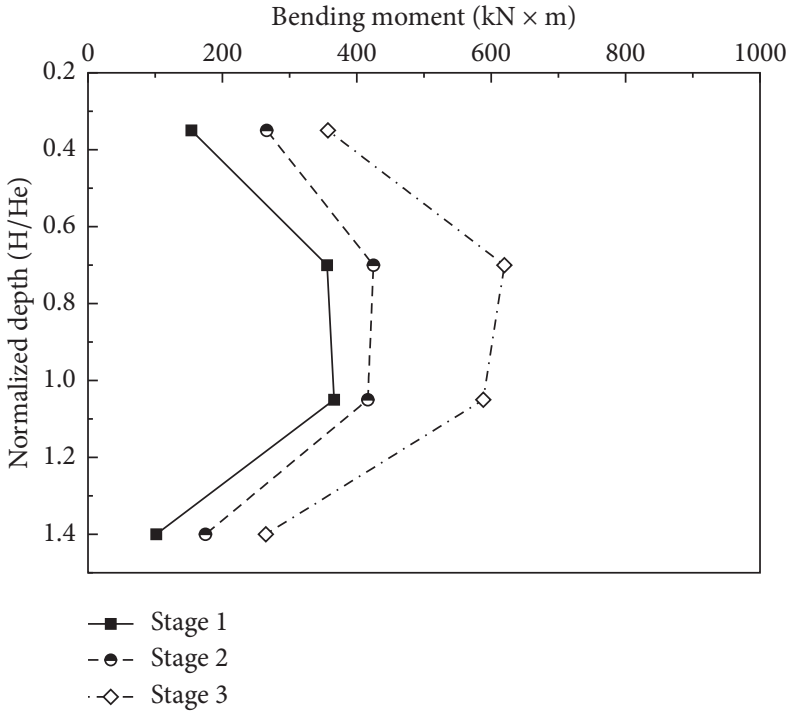

(b)

FiguRE 5: Bending moment curves of the diaphragm walls. (a) Bending moment of the left diaphragm wall. (b) Bending moment of the right diaphragm wall.

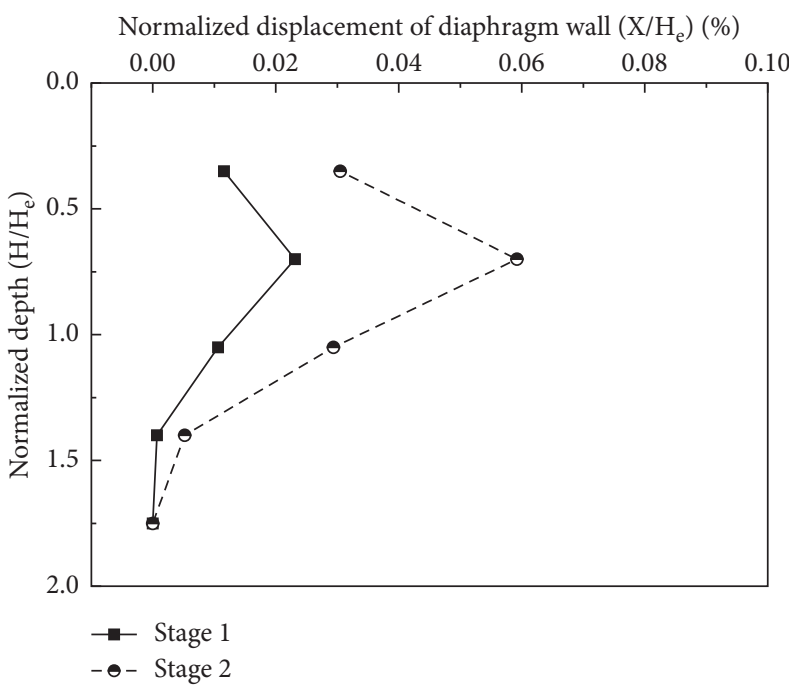

(a)

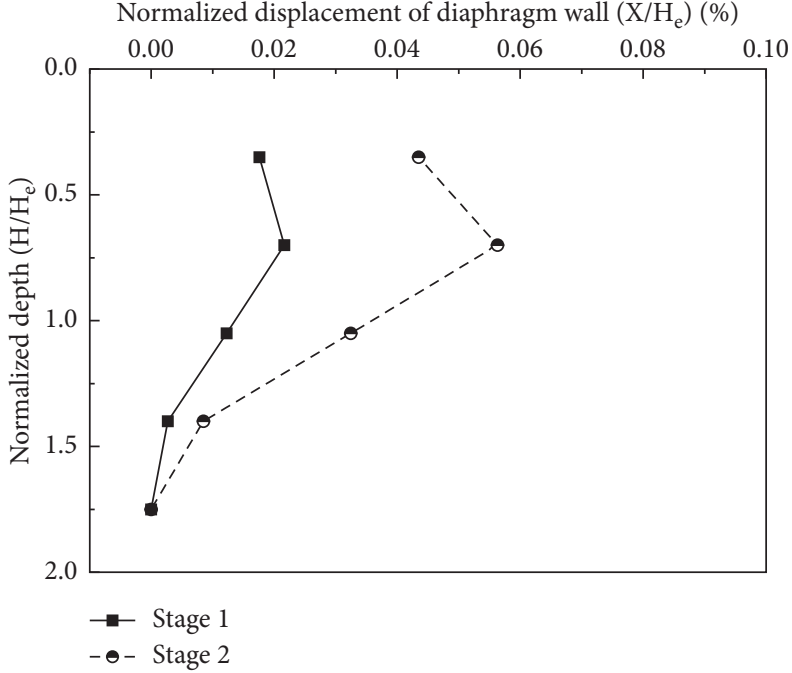

(b)

Figure 6: Horizontal displacement curves of the diaphragm walls. (a) Horizontal displacement of the left diaphragm wall. (b) Horizontal displacement of the right diaphragm wall. 
3.4. The Surface Settlement. In addition to measuring the surface settlement with the laser displacement meters, the surface settlement is also monitored using the Particle Image Velocimetry (PIV) technology based on digital photos. Figure 7 shows the surface settlement curves outside the foundation pit at the different excavation stages, and the result is similar to that in the work of $\mathrm{Ma}$ and $\mathrm{Xu}$ [13]. In the process of the foundation pit excavation, the surface settlement on both sides appears to be the concave groove type. The maximum settlement on both sides of the foundation pit is located about 0.5 times the excavation depth away from the diaphragm wall, and the settlement decreases with the increase in the distance from the diaphragm wall. The surface settlement gradually increases with the increase of the excavation depth during the first and second excavation, whereas the surface settlement rapidly increases after the third excavation. The maximum settlement on the left side of the foundation pit for the third excavation is approximately 5 times higher than that for the second excavation. It is noteworthy that the maximum settlement on the right side of the foundation pit is much smaller than that on the left side, and the tunnel on the right side is just beneath the location of the maximum settlement. It is indicated that the tunnel, which is beneath the location of the maximum settlement, will limit the surface settlement.

Figure 8 compares the experimental data of the surface settlement with the corresponding prediction values obtained from Wang and $\mathrm{Xu}$ [14], where the surface settlement and horizontal distance are normalized in advance. As is shown, the experimental results show good agreements with the prediction values, where the maximum settlements outside the foundation pit are all located about 0.5 times the excavation depth away from the diaphragm wall.

3.5. The Hoop Strain and Earth Pressure of the Tunnels. The distribution of the hoop strain of the tunnels during the foundation pit excavation is shown in Figure 9. The maximum strain of the right tunnel near the foundation pit is about 1.3 times higher than that of the left tunnel, which indicates that the left tunnel far away from the foundation pit is affected less by the foundation pit excavation.

As shown in Figure 9(a), the maximum tensile and compressive strain of the tunnel, which is far away from the foundation pit, appear in the horizontal and vertical directions, respectively [10]. Because the tunnel is far away from the diaphragm wall and the diaphragm wall presents internal convex deformation, the tunnel is mainly affected by the horizontal displacement of soil. The tunnel is vertically compressed and horizontally stretched. The tensile and compressive strain of the tunnel increase with the increase of the excavation depth. The maximum compressive strain on top of the tunnel is 1.2 times higher than that on the bottom of the tunnel, and the tensile strains of the tunnel on the right and left sides are close.
As shown in Figure 9(b), the right tunnel near the foundation pit is still compressed in the vertical direction and stretched in the horizontal direction. The tunnel is close to the diaphragm wall and is greatly affected by excavation unloading. The direction of the maximum tensile strain of the tunnel transforms from $135^{\circ}$ to $315^{\circ}$ for the first and second excavation to $45^{\circ}-225^{\circ}$ for the third excavation. It is suggested that the change in the direction and magnitude of the soil principal stress during the foundation pit excavation would cause the tunnels outside the foundation pit to rotate towards the foundation pit center. The foundation pit excavation causes the displacement and tensile deformation to the adjacent tunnel towards the foundation pit [10]. The displacement and deformation increase as the distance between the tunnel and the foundation pit decreases.

In this section, the change of earth pressure is described by the change rate, which is the ratio of the earth pressure to the initial earth pressure. Due to the breakdown of the earth pressure gauges in the $0^{\circ}$ direction of the left tunnel and in the $0^{\circ}$ and $270^{\circ}$ directions of the right tunnel, only the results obtained by the remaining five earth pressure gauges are analyzed. Figure 10 shows the change rate curves of the earth pressure of the tunnels during the foundation pit excavation. During the excavation, the earth pressure on the bottom of the tunnel slightly changes, while the earth pressure on the left and right sides gradually decreases with the increase of the excavation depth. It is indicated that the soil resistance on both sides of the tunnel is decreased due to the excavation unloading. Thus, the tunnel is vertically compressed and horizontally stretched.

For the left tunnel, the change of earth pressure near the foundation pit is mainly affected by excavation, while the other side is mainly affected by the tunnel. The displacement of soil on the right side of tunnel is greater than that on the left side, and the change rate of the earth pressure at the right side is about 2 times higher than that at the left side. It is indicated that the decrease of the earth pressure near the foundation pit is significantly greater than that far away from the foundation pit during the foundation pit excavation.

The final change rate of the earth pressure at $270^{\circ}$ of the left tunnel is identical to that at $90^{\circ}$ of the right tunnel, and both of them are about $25 \%$. It is indicated that the distance between the tunnel and the foundation pit has little effect on the change rate of the earth pressure at the tunnel side, which is far away from the foundation pit.

3.6. The Horizontal Displacement of the Tunnels. The horizontal displacement of the tunnels is obtained by the PIV. As shown in Figure 11, the horizontal displacement of the tunnels slightly increases after the first and second excavation and then rapidly increases after the third excavation. The maximum horizontal displacement of the right tunnel near the foundation pit is about 1.75 times higher than that of the left tunnel, which is far away from the foundation pit. 


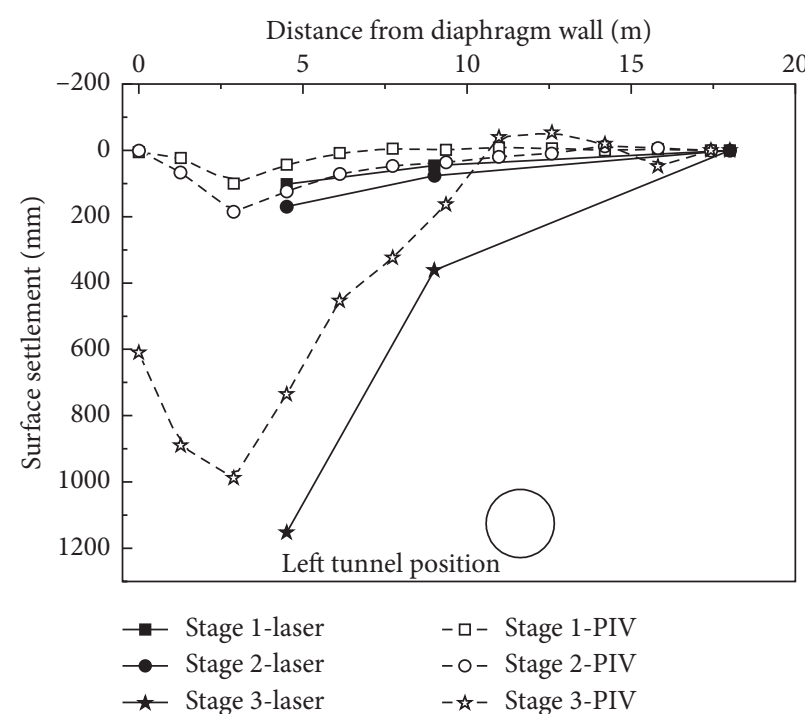

(a)

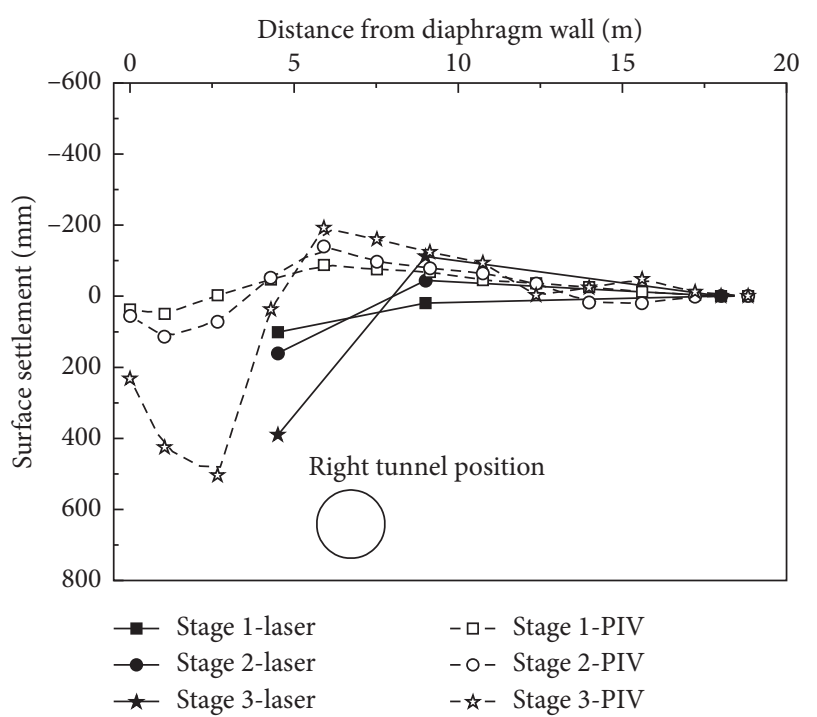

(b)

Figure 7: Surface settlements on the two sides of the foundation pit. (a) Surface settlement on the left side of the pit. (b) Surface settlement on the right side of the pit.

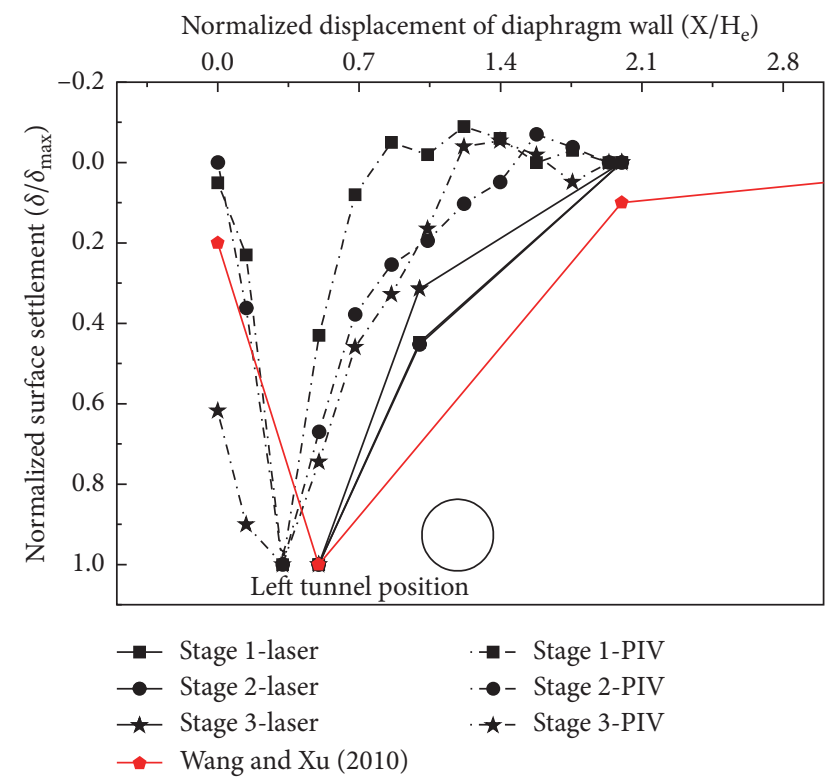

FIGURE 8: Normalization analysis of the surface settlement on the left side of the foundation pit.

\section{Numerical Simulation}

4.1. The Constitutive Model and the Parameters. In this section, the commercial software PLAXIS $2 \mathrm{D}$ is used to simulate the centrifugal model test. In order to eliminate the influence of the model boundary on the numerical results, the boundaries in the numerical model are located beyond 2 times the excavation depth away from the foundation pit. The model range is $100 \mathrm{~m} \times 32 \mathrm{~m}$.

Considering the characteristics of nonlinear unloading and shear hardening of soft soil and the law that shear modulus decays with the strain in small strain stage, the hardening soil model with small strain stiffness (HSS) is chosen as the constitutive model. Due to the instability failure after the third excavation, only the first excavation and second excavation of the foundation pit are simulated. The parameters of the HSS model are listed in Table 3. $c^{\prime}, \varphi^{\prime}$, and the stiffness parameters are obtained from the experiments. The small strain parameters are obtained from the empirical parameters or formulas [15-18].

The numerical model is given in Figure 12, where the foundation pit is $12 \mathrm{~m}$ wide and $9 \mathrm{~m}$ deep. Following the centrifugal test, the foundation pit is excavated in three layers. Only one layer of the inner support is set up on top of the foundation pit. The tunnels with a diameter of $6 \mathrm{~m}$ are buried at the depth of $9 \mathrm{~m}$ and placed at $6 \mathrm{~m}$ and $12 \mathrm{~m}$ away from the diaphragm wall, respectively. The model is undrained, and 15 node units are used.

Figure 13 presents the numerical and experimental horizontal displacement of the left diaphragm after the first and second excavation. As is shown, the numerical results are basically consistent with the corresponding experimental data, which further validates the effectiveness of the proposed numerical model and parameters.

4.2. Effect of the Tunnel Location. The effect of tunnel location on the maximum deformation of the diaphragm wall is shown in Figure 14. The maximum deformation of the diaphragm wall is normalized. $\mathrm{H}$ is the excavation depth at each stage. The maximum deformation of the diaphragm wall slightly increased and then tended to be stable with the increase of the buried depth of the tunnel. The horizontal location of the tunnel has little effect on the maximum deformation of the diaphragm wall. The maximum deformation of the diaphragm wall after the first excavation is about $0.11 \%$ of the excavation depth, while the maximum deformation of the diaphragm wall after the second 


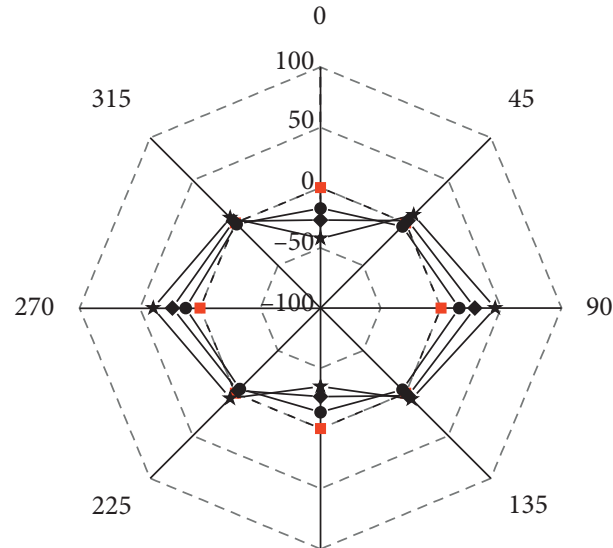

180

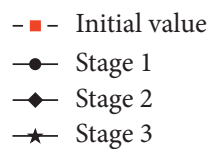

(a)

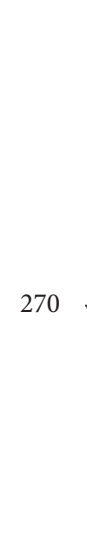

225

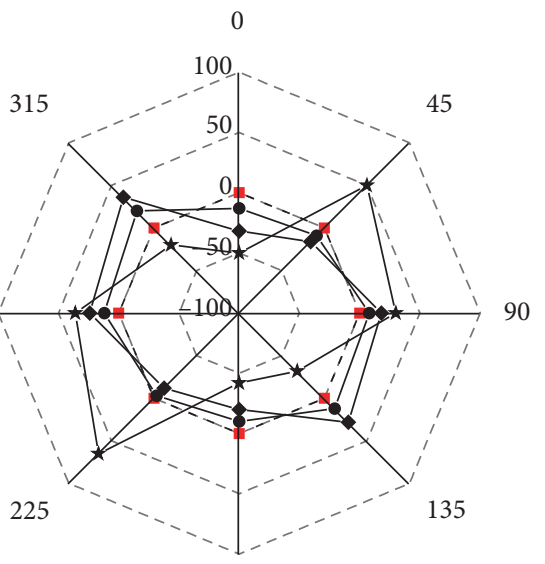

180

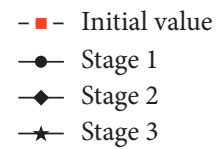

(b)

Figure 9: Hoop strain of the tunnels. (a) Hoop strain of the left tunnel. (b) Hoop strain of the right tunnel.

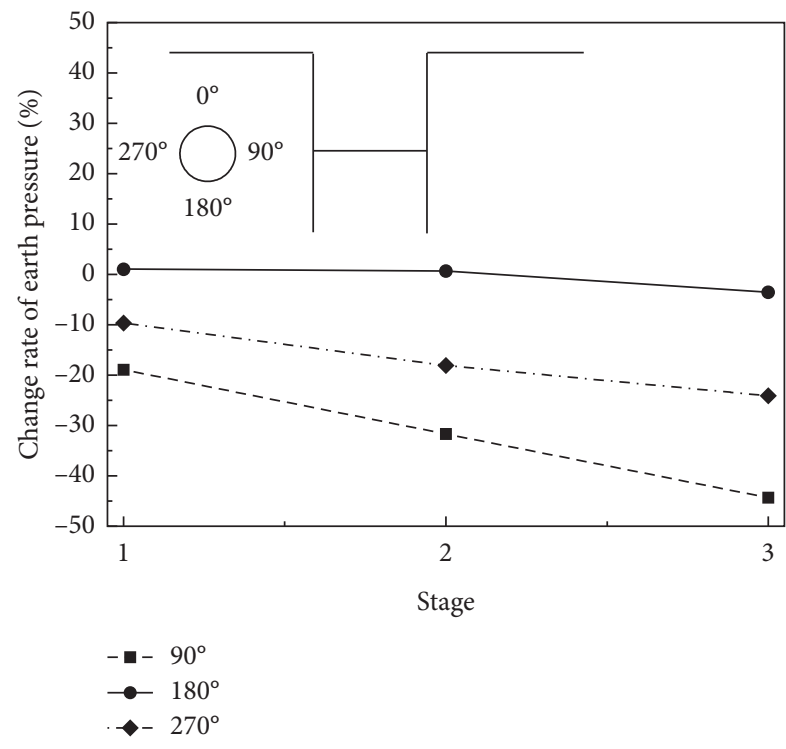

(a)

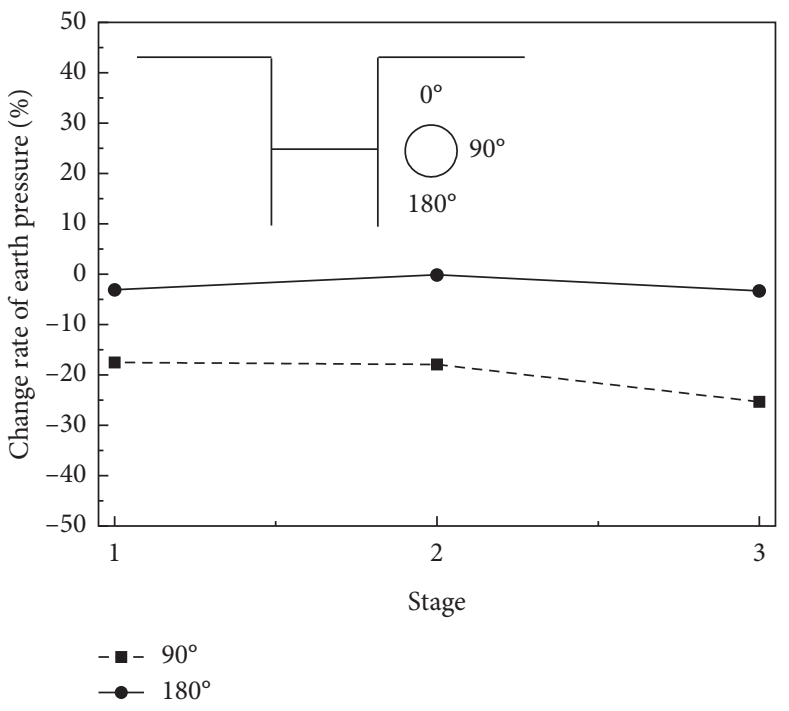

(b)

Figure 10: Change rate of the earth pressure of the tunnels. (a) Change rate of the earth pressure of the left tunnel. (b) Change rate of the earth pressure of the right tunnel.

excavation is about $0.08 \%$ of the excavation depth. Although the existence of the tunnel will change the mechanical characteristics of the foundation to some extent, it has little effect on the diaphragm wall.

Figure 15 shows the effect of the horizontal location and buried depth of the tunnel on the maximum displacement of the tunnel. The maximum displacement of the tunnel is normalized. With the increase of the distance between the tunnel and the diaphragm wall, the influence of excavation unloading decreases gradually. As is shown, the normalized tunnel displacement decreases linearly with the distance between the tunnel and the diaphragm wall but has little relation with the excavation depth. The tunnel displacement firstly increases and then decreases with the increase of the buried depth of the tunnel. After the first excavation, the maximum displacement of the tunnel is $0.058 \%$ of the excavation depth, when the buried depth of the tunnel is $5 \mathrm{~m}$. After the second excavation, the maximum displacement of the tunnel is $0.05 \%$ of the excavation depth, when the buried depth of the tunnel is $7 \mathrm{~m}$. The diaphragm wall presents convex deformation. Because the distance between the tunnel and the diaphragm wall is close, it is considered that 


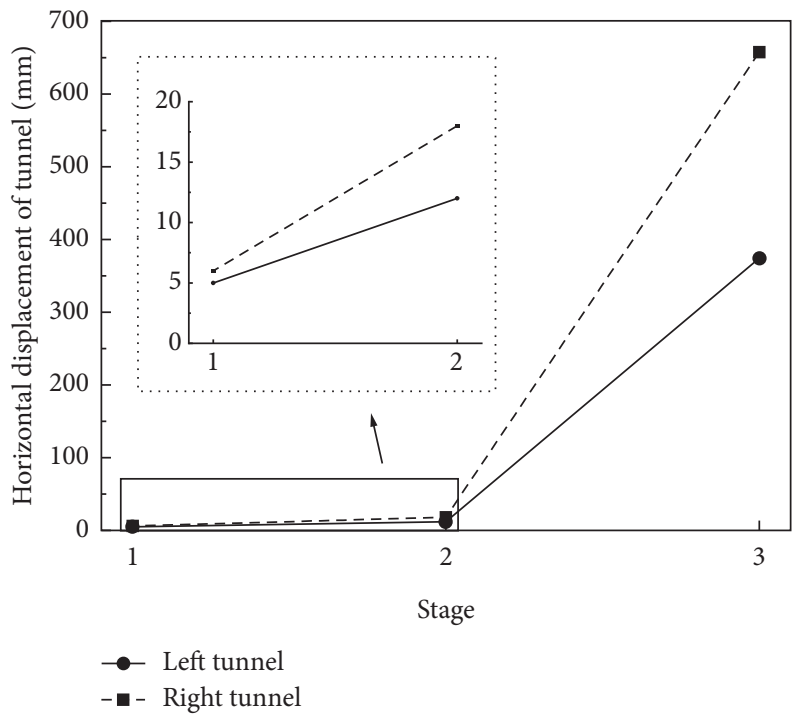

Figure 11: Horizontal displacement of the tunnels.

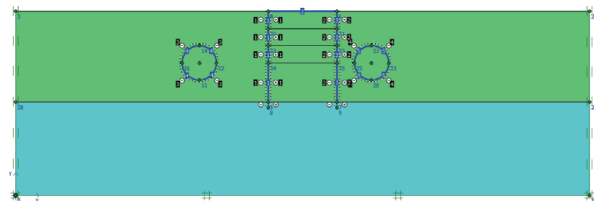

Figure 12: Finite element analysis model.

Table 3: Parameters of the HSS model.

\begin{tabular}{lc}
\hline Parameters & Values \\
\hline$c^{\prime}(\mathrm{kPa})$ & 2.67 \\
$\varphi^{\prime}\left({ }^{\circ}\right)$ & $23^{\circ}$ \\
$P^{\text {ref }}(\mathrm{kPa})$ & $100^{(20)}$ \\
$\psi$ & $0^{(20)}$ \\
$M$ & $0.8^{(21)}$ \\
$v_{u r}$ & $0.2^{(20)}$ \\
$\mathrm{R}_{\mathrm{f}}$ & $0.9^{(22)}$ \\
$E_{\text {oed }}^{\text {ref }}(\mathrm{kPa})$ & 2200 \\
$E_{50}^{\text {ref }}(\mathrm{kPa})$ & 3190 \\
$E_{u r}^{\text {ref }}(\mathrm{kPa})$ & 20030 \\
$G_{0}^{\text {ref }}(\mathrm{kPa})$ & $80120^{(23)}$ \\
$\gamma_{0.7}$ & $7.8 \times 10^{-4(20)}$ \\
\hline
\end{tabular}

the tunnel is affected by the deformation of the diaphragm wall [10]. As the excavation proceeds, the depth of the tunnel with the maximum displacement is gradually downward, which is basically consistent with the location of the maximum deformation of the diaphragm wall. Therefore, more

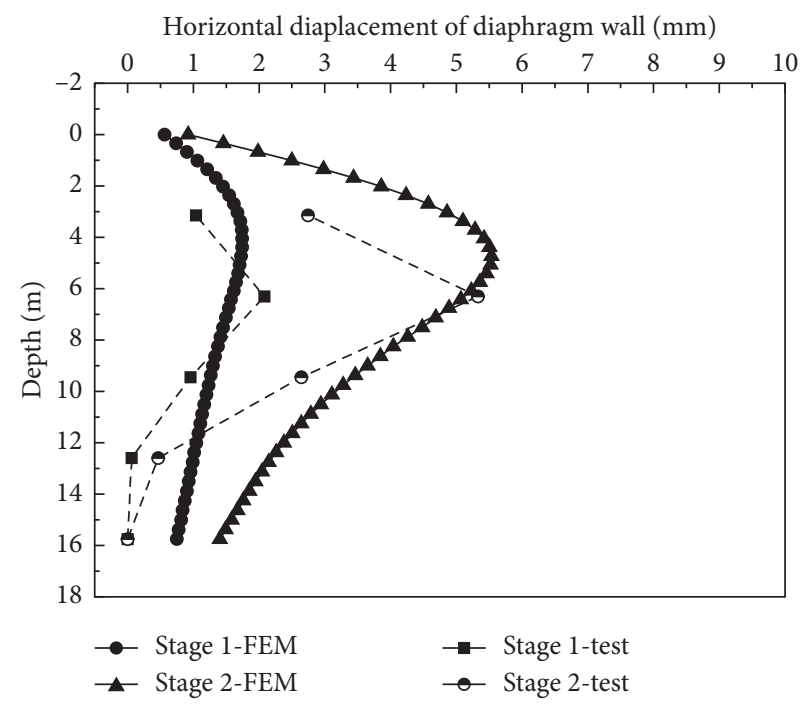

FIgURE 13: Comparison of the presented numerical solution of the left diaphragm wall deformation with experimental data. 


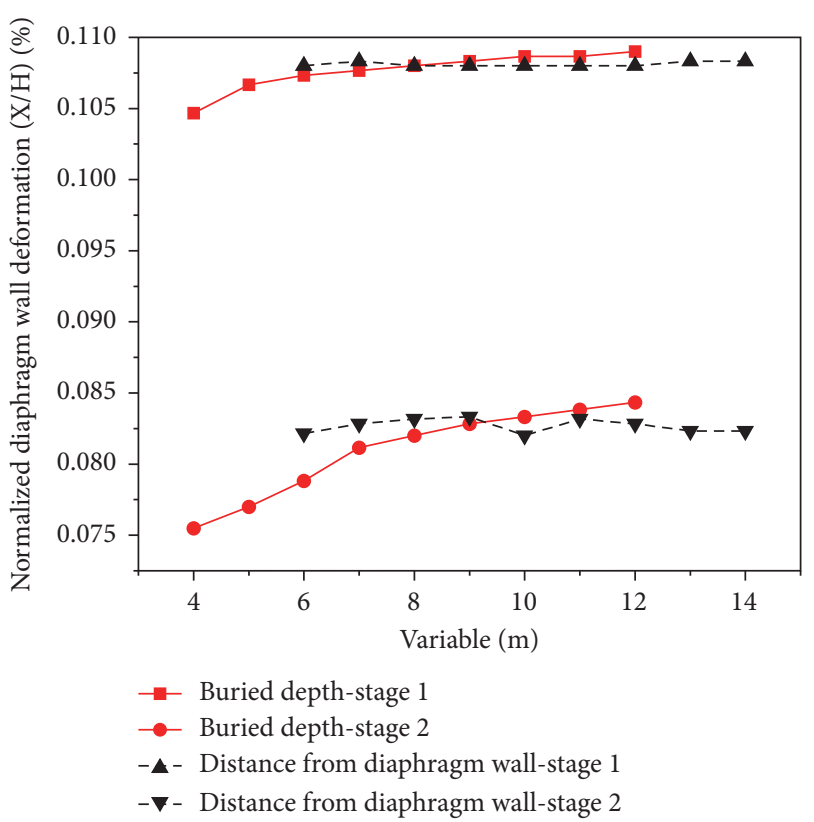

FIGURE 14: Effect of the tunnel location on the maximum deformation of the diaphragm wall.

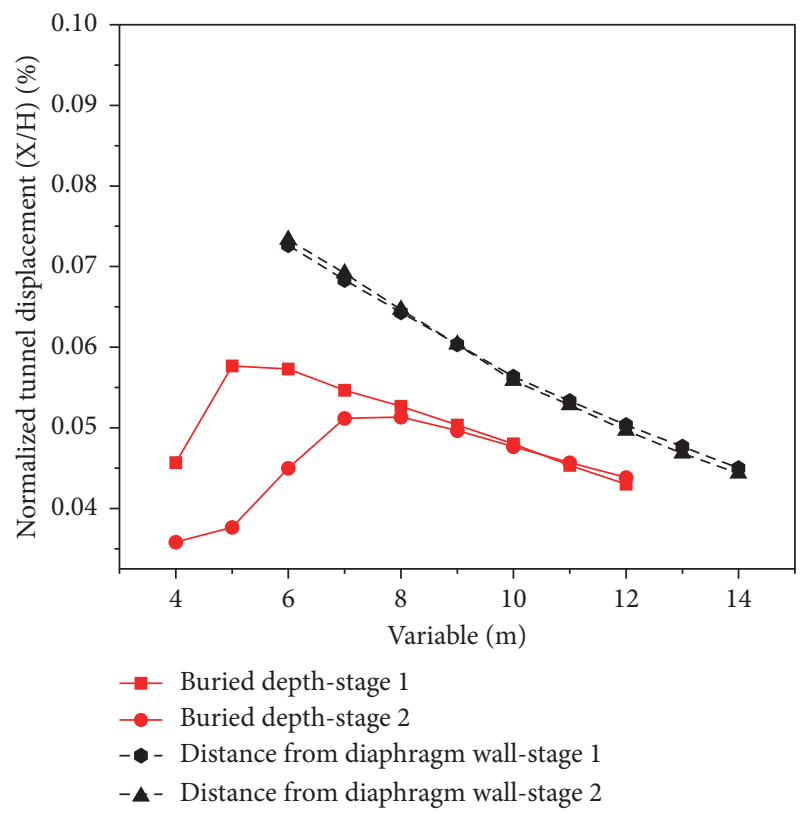

Figure 15: Effect of the tunnel location on the maximum displacement of the tunnel.

attention should be paid to the tunnel located near the maximum deformation of the diaphragm wall during construction.

\section{Conclusions}

Based on the centrifugal model test and numerical model, the influence of the foundation pit excavation on the adjacent tunnels at different locations in soft soil is investigated. The main conclusions can be drawn as follows:
(1) During the stable stage of the foundation pit excavation in soft soil, the bending moment and horizontal displacement of the diaphragm walls on the left and right sides approach each other, and the diaphragm walls present convex deformation towards foundation pit. The surface settlement outside the diaphragm wall on both sides appears to be the concave groove type, and the maximum settlement on both sides of the foundation pit is located about 0.5 times the excavation depth away from the diaphragm wall. The decrease of the earth pressure near the foundation pit is significantly greater than that far away from the foundation pit during the foundation pit excavation.

(2) After the third excavation, the foundation pit loses stability, and the shear bands are nearly tangent to the tunnels. The maximum bending moment of the diaphragm wall far away from the tunnel is located at the bottom of the diaphragm wall and significantly larger than that of the diaphragm wall near the tunnel. The left tunnel, which is far away from the foundation pit, is vertically compressed and horizontally stretched, while the maximum tensile strain of the right tunnel near the foundation pit is in the direction of $45^{\circ}-225^{\circ}$. The displacement of the tunnels on both sides rapidly increases, and the displacement of the right tunnel near the foundation pit is larger than that of the left tunnel, which is far away from the foundation pit. The deformation of the diaphragm wall and the surface settlement are limited by the existing tunnel.

(3) The maximum deformation of the diaphragm wall slightly increased and then tended to be stable with the increase of the buried depth of the tunnel. The horizontal location of the tunnel has little effect on the maximum deformation of the diaphragm wall. The maximum displacement of the tunnel almost linearly decreases with the increase of the horizontal distance between the tunnel and the diaphragm wall and firstly increases and then decreases with the increase of the buried depth of the tunnel. The deformation of diaphragm wall will affect the tunnel displacement to some extent.

\section{Data Availability}

All the data used to support the findings of this study are included within the article.

\section{Conflicts of Interest}

The authors declare that there are no conflicts of interest regarding the publication of this paper.

\section{Acknowledgments}

The authors thank the National Key Research and Development Program of China (no. 2016YFC0800200), the National Natural Science Foundation of China (Grant no. 
41702329), the Zhejiang Provincial Natural Science Foundation (nos. LQ18E080001 and LY19E080013), the project supported by the Foundation of Key Laboratory of Soft Soils and Geoenvironmental Engineering (Zhejiang University), the Ministry of Education (2019P02), and the Ningbo City Science and Technology Project for Public Benefit (2019C50014) for the financial support of this study.

\section{References}

[1] F.-Y. Meng, R.-P. Chen, H.-N. Wu, S.-W. Xie, and Y. Liu, "Observed behaviors of a long and deep excavation and collinear underlying tunnels in Shenzhen granite residual soil," Tunnelling and Underground Space Technology, vol. 103, p. 103504, 2020.

[2] G. Y. Gao, M. Gao, C. B. Yang, and Z. S. Yu, "Influence of deep excavation on deformation of operating metro tunnels and countermeasures," Chinese Journal of Geotechnical Engineering, vol. 32, no. 3, pp. 453-459, 2010, in Chinese.

[3] G. Zheng and S.-W. Wei, "Numerical analyses of influence of overlying pit excavation on existing tunnels," Journal of Central South University of Technology, vol. 15, no. S2, pp. 69-75, 2008.

[4] X. Huang, H. F. Schweiger, and H. Huang, "Influence of deep excavations on nearby existing tunnels," International Journal of Geomechanics, vol. 13, no. 2, pp. 170-180, 2013.

[5] C. W. W. Ng, J. Shi, D. Mašín, H. Sun, and G. H. Lei, "Influence of sand density and retaining wall stiffness on threedimensional responses of tunnel to basement excavation," Canadian Geotechnical Journal, vol. 52, no. 11, pp. 1811-1829, 2015.

[6] C. W. W. Ng, J. Shi, and Y. Hong, "Three-dimensional centrifuge modelling of basement excavation effects on an existing tunnel in dry sand," Canadian Geotechnical Journal, vol. 50, no. 8, pp. 874-888, 2013.

[7] G. Zheng, S. W. Wei, S. Y. Peng, Y. Diao, and C. W. W. Ng, "Centrifuge modeling of the influence of basement excavation on existing tunnels," in Proceedings of the 7th International Conference on Physical Modelling in Geotechnics 2010, ICPMG, p. 523, Zurich, Switzerland, June 2010.

[8] J. Cui, J. Li, L. Li, and G. Zhao, "Deformation of subway tunnels affected by adjacent excavation: in-situ monitoring and centrifugal model test," in Proceedings of the GeoShanghai International Conference, pp. 429-438, Shanghai, China, May 2018.

[9] R. P. Chen, A. Al-Madhagi, and F. Meng, “Three-dimensional centrifuge modeling of influence of nearby excavations on existing tunnels and effects of cut-off walls," Chinese Journal of Geotechnical Engineering, vol. 40, no. S2, pp. 6-11, 2018, in Chinese.

[10] G. Zheng, Y. DU, X. Cheng, Y. Diao, X. Deng, and F. Wang, "Characteristics and prediction methods for tunnel deformations induced by excavations," Geomechanics and Engineering, vol. 12, no. 3, pp. 361-397, 2017.

[11] H. H. Zhang, Centrifugal Model Test Study on Excavation of Ultra-deep Foundation Pit with Soft Soil, Tongji University, Shanghai, China, 2009, in Chinese.

[12] Q. Xu, X. Ma, H. Zhu, and X. Ge, "Centrifuge study on ultradeep foundation pit excavation in soft ground," in Proceedings of the GeoShanghai International Conference, pp. 292-299, Shanghai, China, June 2010.

[13] X. Ma and J. Xu, "Centrifuge model tests on excavation in Shanghai clay using in-flight excavation tools," in Proceedings of the 9th International Conference on Physical Modelling in Geotechnics, pp. 419-423, London, UK, July 2018.

[14] W. D. Wang and Z. H. Xu, "Simplified analysis method for evaluating excavation-induced damage of adjacent buildings," Chinese Journal of Geotechnical Engineering, vol. 32, no. S1, pp. 32-38, 2010, in Chinese.

[15] R. B. J. Brinkgreve, S. Kumarswamy, and W. M. Swolfs, Plaxis Material Models Manual, Delft University of Technology \& PLAXISB, The Netherlands, 2006.

[16] T. S. Nagaraj, N. Miura, S. G. Chung, and K. Nagendra Prasad, "Analysis and assessment of sampling disturbance of soft sensitive clays,” Géotechnique, vol. 53, no. 7, pp. 679-683, 2003.

[17] H. Schweiger, "Design of deep excavations with FEM-Influence of constitutive model and comparison of EC7 design approaches," Earth Retention Conference, vol. 3, pp. 804-817, 2010.

[18] W. Zhang, A. T. C. Goh, and F. Xuan, "A simple prediction model for wall deflection caused by braced excavation in clays," Computers and Geotechnics, vol. 63, pp. 67-72, 2015. 